\title{
Standardizing Pancreas Research
}

\author{
Martin A. Makary, MD, MPH \\ Department of Surgery, Johns Hopkins University School of Medicine, Baltimore, MA
}

This report highlights a subject that has received little attention to date: standardization of surgery in cancer research. For too long, medical oncologists and surgeons have designed trials in isolation from each other. As the authors point out, a hazard to such a partisan approach is the lack of attention to detail in the field least familiar to the commanding investigators. In this case, the lack of attention to surgical details in ACOSOG Z5031 resulted in an unknown influence of confounding variables. Because these variables affect the true margin status and because they were not accounted for, the overall study results are challenging to interpret and difficult to apply broadly. Surgery remains the only cure for pancreas cancer, and chemotherapy remains the only hope for many patients. Developing research protocols that ensure a complete and standard measurement of medical and surgical therapy is critical to interpreting data and making generalizable conclusions.

More specifically, the authors point out that ACOSOG Z5031 failed to adopt common standards for both the surgical removal of the specimen and the analysis of the margin and lymph node status in the pathology laboratory. They point out that the margin status among enrolled patients could have been misclassified or altered as a result of the lack of standard principles in removing and analyzing the pancreatic head from the area of the superior mesenteric artery (a difficult part of the Whipple operation that is known to have high variability in technique). Most concerning was the finding that the number of lymph nodes evaluated in ACOSOG Z5031 ranged from 1 to 49 even though $73 \%$ of patients had lymph node involvement of the cancer. Definitions and guidelines from the American Joint

(C) Society of Surgical Oncology 2010

Published Online: 23 November 2010

M. A. Makary, MD, MPH

e-mail: mmakary1@jhmi.edu
Committee on Cancer and the College of American Pathologists have been established to address such important variations in surgical technique and tissue analysis. For such a key national trial, failure to use these guidelines detracts from the generalizability of the results. Importantly, the lack of standardization could have been prevented with good preplanning during the design.

Another source of variability peripherally mentioned in this report is how surgeons handle tumors that are slightly adherent to the superior mesenteric vein or portal vein. Commonly, some surgeons first attempt to dissect ("scrape") it off the vein and then ask the pathologist to look for cancer at the vein margin before deciding whether to perform a vein resection; other surgeons use a more planned vein resection approach. Although judgment is required in this situation, these two styles in approaching tumors slightly adherent to the vein should also be standardized because an en-bloc resection is more oncologically appropriate and a lack of standardization in frozen-section analyses of the vein margin could be associated with false-negative findings. Beyond the techniques which Katz et al. outlined, a standardized approach to vein involvement should also be addressed in pancreas cancer trials.

Although Katz et al. appropriately identify holes in the study design of ACOSOG Z5031, a few of the highlighted errors of omission are more symbolic than clinically important. A few such errors may simply represent an absence in documentation-a common bias in quality research. For example, Katz et al. report that the presence or absence of a visible pancreatic mass was noted in the operative report of just $76 \%$ of the patients, yet we know from the study inclusion criteria and pathology reports that all patients clearly had a mass. The authors are also critical of variability in surgeon's documentation of estimated blood loss, an important clinical data point that surgeons should routinely copy from the anesthesia record to the operative report. However, this surrogate variable has no effect on cancer survival. Finally, the authors criticize the 
surgeon's in the study for only identifying an intraoperative stage in $13 \%$ of patients, yet it is standard practice to explore the abdomen for suspicious lesions as the first step in performing a Whipple operation, and many surgeons rely on the more accurate final pathology report for clinically meaningful tumor, node, metastasis system staging information. Did $87 \%$ of patients have their surgeon forget to look for precluding advanced stage disease at the beginning of the Whipple operation? Although the surgeons should have documented the intraoperative tumor, node, metastasis system stage observed for completeness, it is likely that the $87 \%$ noncompliance rate was the noncompliance rate for the appropriate documentation, not for performing this critical step. However, the authors are right to scrutinize attention to detail in a historic cancer trial. Moreover, lack of attention to detail in documentation may be a surrogate of wider variation in surgical practice style not accounted for in the data collected. To better inform our medical community based on our experience in cancer care, we as surgeons should adopt standardized templates for common operations, allowing for like comparisons. This practice is most critical when trials are being conducted that could affect thousands of future patients.

The poor quality control in surgical therapy observed in ACOSOG Z5031 represents a larger problem in American medicine: the lack of standardized health care. Furthermore, most problems in any organizational behavior stem from a lack of input from front-line providers. Katz et al. are right to insist on quality-control metrics for surgical care in oncology trials. Attention to surgical and pathological technique could influence margin status and stage, and ultimately change a study's conclusions. The demand to standardize and measure surgical factors is an important development in the century-long improvement of clinical trials through increasingly accounting for the many variables which influence outcome. 